\title{
Rearrangement of Benzyl-type Radical in Corona Discharge of 2,6-Dichlorotoluene
}

\author{
Young Wook Yoon, Seung Woon Lee, and Sang Kuk Lee* \\ Department of Chemistry and The Chemistry Institute for Functional Materials, Pusan National University, Pusan 609-735, \\ Korea.*E-mail: sklee@pusan.ac.kr \\ Received June 3, 2010, Accepted July 11, 2010
}

\begin{abstract}
Using a pinhole-type glass nozzle equipped for a corona-excited supersonic expansion (CESE), precursor 2,6-dichlorotoluene seeded in a large amount of inert carrier gas helium was discharged to produce jet-cooled but electronically excited benzyl-type radicals. The visible vibronic emission spectrum was recorded with a long-path monochromator to observe vibronic bands in the $\mathrm{D}_{1} \rightarrow \mathrm{D}_{0}$ electronic transition of benzyl-type radicals. The spectral analysis revealed the generation of not only the 2,6-dichlorobenzyl radical as a typical product, but also the $o$-chlorobenzyl radical as an unexpected species, which indicates the possible molecular rearrangement in eliminating a chlorine atom from the benzene ring. A possible mechanism is proposed for the formation of the $o$-chlorobenzyl radical from the precurs or in the gas phase.
\end{abstract}

Key Words: Molecular rearrangement, Corona discharge, Chlorobenzyl radical

\section{Introduction}

Transient species such as molecular radicals and ions have long been believed to play an important role as intermediates in chemical reaction. ${ }^{1}$ The characteristics and structure of transient species provide further evidence to define the property of chemical reactivity in the fundamental issue of reaction dynamics. ${ }^{2}$

The benzyl radical, the prototype aromatic radical, has been long believed to be the most important intermediate in aromatic chain reactions and been the subject of many spectroscopic studies. ${ }^{3}$ Several techniques have been developed for the production of benzyl-type radical from precursor, in which the corona discharge using high voltage DC has been recognized to be one of the most convenient methods because of the simple experimental technique. For corona discharge, a simple pinhole-type glass nozzle employed in a corona-excited supersonic expansion system has been proved to be a powerful tool for the production of electronically hot, but jet-cooled benzyl-type radicals from precursors with a large amount of inert carrier gas for observation of vibronic emission spectra. ${ }^{4-10}$

The first halogen-substituted benzyl radical was produced by Bindley et al. from the high voltage electric discharge of the corresponding fluorotoluenes, ${ }^{11}$ in which all three isomers of fluorobenzyl radicals were clearly identified through an analysis of the vibronic spectra. Since then, many different types of fluorine-substituted benzyl-type radicals were confirmed by corona discharge of the precursors. ${ }^{4,12-15}$

Although chlorine-substituted benzyl-type radicals have been recognized to emit much weaker fluorescence in the electronic transition, chlorobenzyl radicals have been investigated through vibronic emission spectra observed with $\mathrm{CESE}^{16-18}$ and laserinduced fluorescence techniques. ${ }^{19}$ However, the study on multi-chlorine substituted benzyl radicals has remained very limited because of their characteristics of weak intensity with increasing number of chlorine atoms in the benzene ring. Up to date, the 2,6-dichlorobenzyl radical is the only multi-chlorine substituted benzyl radical of which vibronic spectra have been successfully observed by CESE technique. ${ }^{20}$

In this paper, we present the evidence of unexpected observation of the $o$-chlorobenzyl radical during the corona discharge of 2,6-dichlorotoluene with a large amount of inert carrier gas helium in the CESE system, for which a possible mechanism is proposed to explain the formation of the unexpected product in corona discharge.

\section{Experimental}

The experimental setup employed in this work is similar to that described elsewhere. ${ }^{21,22}$ It consists of a pinhole-type glass nozzle equipped with a CESE system for the formation and excitation of radicals in a jet from a precursor, and a monochromator for observation of the vibronic emission spectra.

The vapor of the precursor 2,6-dichlorotoluene (Aldrich, reagent grade) heated to $50{ }^{\circ} \mathrm{C}$ was mixed with 2.0 atm of inert carrier gas $\mathrm{He}$, in which the precursor concentration in the gas mixture was adjusted to obtain the maximum intensity of fluorescence monitored from the strongest band. In order to increase the stability of the corona discharge inside nozzle, we adapted a modified pinhole-type glass nozzle with an external heating device. The heating of the nozzle significantly improved the stability of the discharge by preventing condensation of the precursor inside the nozzle. A discharging voltage of approximately $1.5 \mathrm{kV}$ with a current of less than $5 \mathrm{~mA}$ was applied to observe not only the maximum emission intensity from the strongest band but also the minimum production of small fragments such as $\mathrm{C}_{2}$ and $\mathrm{CH}$ radicals that show strong emission in the spectral region of benzyl-type radicals.

The Pyrex expansion chamber was evacuated by a mechanical vacuum pump, providing a chamber pressure of about 1.0 Torr during the continuous jet expansion with a $0.5 \mathrm{~mm}$ diameter orifice and a backing pressure of $2.0 \mathrm{~atm}$, which was largely limited by the tolerance of the glass materials used for the nozzle. With the corona discharge of the precursor, a blue- 
green jet appeared, indicating the production of benzyl-type radicals. The emission from the jet area at a diameter of $4 \mathrm{~mm}$ below the nozzle throat was collected using a quartz lens $(\mathrm{D}=$ $38 \mathrm{~mm}, \mathrm{~F}=50 \mathrm{~mm}$ ) placed inside the chamber and focused on the slit of a long-path monochromator (Jobin Yvon U1000) equipped with a cooled PMT (Hamamatsu R649). The visible vibronic emission spectrum was recorded by scanning from 18000 to $22500 \mathrm{~cm}^{-1}$ at steps of $2.0 \mathrm{~cm}^{-1}$ with a slit width of $200 \mu \mathrm{m}$ over $1 \mathrm{hr}$. The wavenumber of the spectra was calibrated by the He atomic lines observed at the same time with the spectra and believed to be accurate within $\pm 1.0 \mathrm{~cm}^{-1}$. 23

\section{Results and Discussion}

It has been confirmed that a well-controlled corona discharge of substituted toluene with a large amount of carrier gas in jet expansion produces corresponding benzyl-type radicals in vibronically excited states. ${ }^{24}$ Although the mechanism for the formation and excitation of benzyl-type radicals in CESE has not been exactly established, it has been suggested that when He is employed as the carrier gas, the metastable He atom in the $1 \mathrm{~s} 2 \mathrm{~s}$ ${ }^{3} \mathrm{~S}_{1}$ state that is initially excited by the corona discharge transfers its excess energy to the precursor through a collisional process. ${ }^{25}$ The collisional energy transfer from metastable He to other species is a well-known process in the excitation of the $\mathrm{Ne}$ atoms for the HeNe laser. The electronically excited precursors then decomposes to produce corresponding benzyltype radicals by losing a hydrogen atom from the vulnerable methyl group rather than from the benzene ring, as evidently observed in the CESE system. The hydrogen atom loosely held in the $\mathrm{C}-\mathrm{H}$ bond of the methyl group at the excited electronic state is easily eliminated to form the benzyl-type radical. ${ }^{26}$

The molecular radicals produced in corona excitation may undergo collisional relaxation with He atoms, producing electronically hot but jet-cooled species by removing the rotational

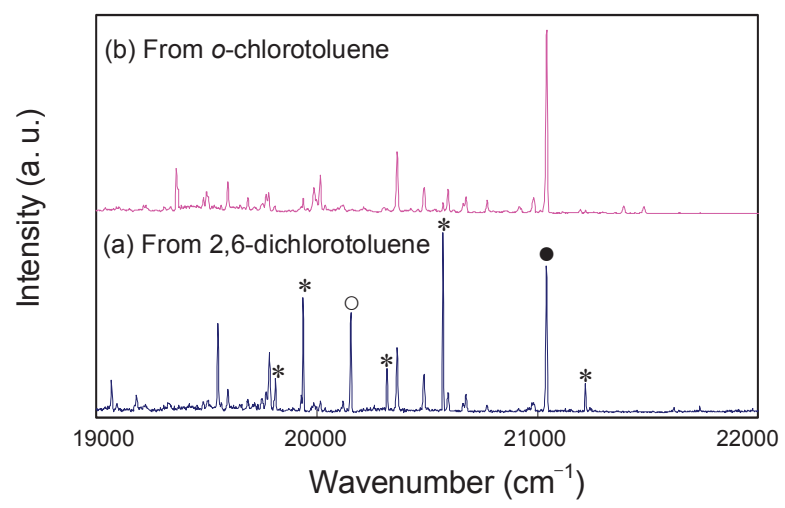

Figure 1. A portion of the vibronic emission spectra observed from the corona discharge of precursors (a) 2,6-dichlorotoluene and (b) o-chlorotoluene with a large amount of inert carrier gas helium in the CESE system. The closed $(\bullet)$ and open ( $\circ$ ) circles represent the origin bands of the $o$-chlorobenzyl and 2,6-dichlorobenzyl radicals, respectively. The spectrum (a) exhibits the bands originating from the $o$-chlorobenzyl, as well as the 2,6-dichlorobenzyl radicals, while the spectrum (b) shows the bands belonging to the $o$-chlorobenzyl radicals only. Atomic lines denoted by an asterisk $(*)$ show strong intensity in the spectrum (a). and vibrational energy in the excited electronic states. Contour simulation using Boltzmann distribution ${ }^{27}$ showed a rotational temperature of approximately $40 \mathrm{~K}$ for the benzyl-type radicals generated in the CESE system, which is believed to be relatively high for species in supersonic jet expansion because the radicals were produced under the condition of high voltage discharge, low backing pressure, and continuous expansion.

Since the efficient vibronic coupling between two excited electronic states transfers the population from $\mathrm{D}_{2}$ to $\mathrm{D}_{1}$ states, the benzyl-type radicals emit the fluorescence in the $\mathrm{D}_{1} \rightarrow \mathrm{D}_{0}$ electronic transition, in which the origin band typically exhibits the strongest intensity at the highest wavenumber. Thus, the spacing between the origin band and other vibronic bands represents the vibrational mode frequencies in the ground electronic state, indicating that the vibronic emission spectrum observed in the CESE system should be similar to the LIF-DF spectra while pumping the origin band of the electronic transition. ${ }^{4}$

In Fig. 1, the spectra (a) and (b) show the visible vibronic emission spectra observed from the corona discharge of precursors 2,6-dichlorotoluene and o-chlorotoluene, respectively, seeded in a large amount of an inert carrier gas He using a pinholetype glass nozzle in the CESE system. The spectrum (b) represents the evidence of the $o$-chlorobenzyl radical in the $\mathrm{D}_{1} \rightarrow$ $\mathrm{D}_{0}$ transition since the very strong origin band at $21040 \mathrm{~cm}^{-1}$ and other vibronic bands show an excellent agreement with those previously reported for the $o$-chlorobenzyl radical. ${ }^{18}$

Because the precursor was employed to produce the 2,6dichlorobenzyl radical in the corona excitation as the standard procedure, we searched for evidence of the formation of the 2,6-dichlorobenzyl radical in the spectrum (a). The band at $20154 \mathrm{~cm}^{-1}$ with a very strong intensity coincides with the origin band in the $\mathrm{D}_{1} \rightarrow \mathrm{D}_{0}$ transition $^{20}$ of the 2,6-dichlorobenzyl radical, confirming the production of the radical by dissociation of one of the $\mathrm{C}-\mathrm{H}$ bonds of the methyl group at the excited electronic state. The elimination mechanism of the hydrogen atom from the methyl group has been described for $o$-xylene in the time-resolved experiment, in which the excitation of molecules from $\mathrm{S}_{0}$ to $\mathrm{S}_{1}$ states may loosen the $\mathrm{C}-\mathrm{H}$ bond and then be dissociated to produce the benzyl-type radical. ${ }^{26}$

Besides the bands belonging to the 2,6-dichlorobenzyl radical, we observed many strong bands in the blue region of the origin band. In order to identify the origin of these bands, we compared them with those obtained under the same experimental condition from the corona discharge of precursor $O$-chlorotoluene. From the comparison, many vibronic bands, including the strongest one at $21040 \mathrm{~cm}^{-1}$ in the spectrum (a), showed an excellent agreement with those in the spectrum (b), confirming that the $o$-chlorobenzyl radical is formed from the precursor 2,6-dichlorotoluene in corona discharge.

The formation of the $o$-chlorobenzyl radical from the precursor 2,6-dichlorotoluene in the corona discharge indicates the existence of another pathway in producing the benzyl-type radical from the precursor, in addition to the simple loosening of the $\mathrm{C}-\mathrm{H}$ bond in the excited electronic state. The simple mechanism for formation of benzyl-type radical is to dissociate a hydrogen atom from a methyl group. We assume there exist two possible pathways in the formation of the $o$-chlorobenzyl radical 


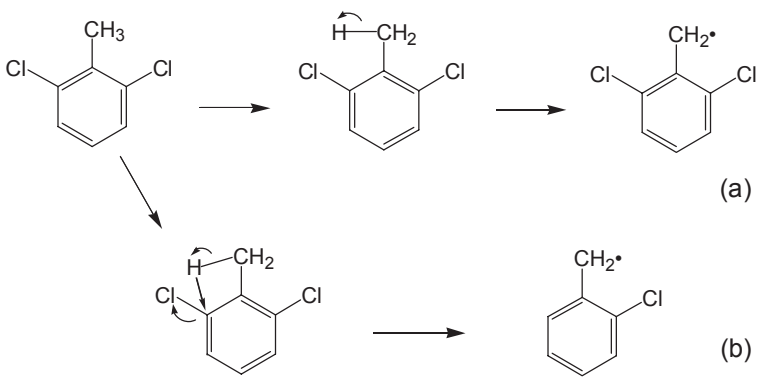

Figure 2. Scheme for the formation of the $o$-chlorobenzyl and the 2,6dichlorobenzyl radicals from 2,6-dichlorotoluene in the gas phase. A loose $\mathrm{C}-\mathrm{H}$ bond of a methyl group dissociates to produce (a) the 2,6dichlorobenzyl radical, while a chlorine atom is replaced by a hydrogen atom attacking a carbon atom at the $o$-position to produce (b) the $o$ chlorobenzyl radical.

Table 1. List of the vibronic bands observed and their assignments

\begin{tabular}{ccc}
\hline position $^{a}$ & intensity & assignments \\
\hline 21216 & $\mathrm{~m}$ & He atomic \\
21040 & vs & Origin of $o$-isomer \\
20986 & vw & \\
20674 & $\mathrm{~m}$ & $9 \mathrm{~b}_{1}^{0}$ of $o$-isomer \\
20594 & $\mathrm{~m}$ & $7 \mathrm{a}_{1}^{0}$ of $o$-isomer \\
20572 & vs & H atomic \\
20484 & $\mathrm{~m}$ & $6 \mathrm{~b}_{1}^{0}$ of $o$-isomer \\
20362 & $\mathrm{~s}$ & $6 \mathrm{a}_{1}^{0}$ of $o$-isomer \\
20318 & $\mathrm{~s}$ & He atomic \\
20154 & vs & Origin of $2,6-$ \\
20118 & vw & $9 \mathrm{~b}_{1}^{0} 6 \mathrm{~b}_{1}^{0}$ of $o$-isomer \\
20014 & vw & $1_{1}^{0}$ of $o$-isomer \\
19986 & vw & $18 \mathrm{a}_{1}^{0}$ of $o$-isomer \\
19938 & vs & He atomic \\
19930 & w & $9 \mathrm{a}_{1}^{0}$ of $2,6-$ \\
19810 & $\mathrm{~m}$ & He atomic \\
19784 & $\mathrm{~m}$ & $7 \mathrm{a}_{1}^{0}$ of $2,6-$ \\
19596 & $\mathrm{w}$ & $19 \mathrm{a}_{1}^{0}$ of $o$-isomer \\
19552 & $12_{1}^{0}$ of $2,6-$ \\
19182 & $\mathrm{~s}$ & $\mathrm{a}_{1}^{0} 12_{1}^{0}$ of $2,6-6-$ \\
19070 & & \\
\hline
\end{tabular}

${ }^{a}$ Measured in air $\left(\mathrm{cm}^{-1}\right)$.

from 2,6-dichlorotoluene: The first one is a two-step reaction in which the precursor loses a hydrogen atom from a methyl group to produce the 2,6-dichlorobenzyl radical, after which a chlorine atom is replaced by a hydrogen atom at the ortho position, resulting in the generation of the $o$-chlorobenzyl radical. However, the removal of a chlorine atom by a hydrogen atom via intermolecular collision is not a feasible process in the gas phase reaction because the steric factor of the collision theory is very small in a large aromatic reaction. ${ }^{28}$ The second pathway is the direct replacement of a chlorine atom by a hydrogen atom of a methyl group through intramolecular reaction. The weakness of the $\mathrm{C}-\mathrm{H}$ bond may let the hydrogen atom attack the atom at the nearest position, in the ortho position of the benzene ring, as described in the mechanism presented in Fig. 2.

The weak observation of the 2,6-dichlorobenzyl radical in the spectrum (a) may attribute to less stability and low quantum yield of the dichlorobenzyl radicals. It has been well recognized that the substitution of heavy halogen elements such as chlorine and bromine reduce dramatically the emission intensity, making it very difficult to observe the emission spectrum in the electronic transition. The 2,6-dichlorobenzyl radical is the only species that has been reported among bi-substituted chlorobenzyl radicals, even though many kinds of multi-fluorine substituted benzyl radical have been successfully observed in emission spectra.

Also, the observation of strong atomic lines in the spectrum (a) indicates that there are the excess amount of metastable atomic species compared to that of the precursor because the precursor 2,6-dichlorotoluene has a lower vapor pressure than that of $o$-chlorotoluene at the room temperature. The vibronic bands observed in the spectrum (a) are assigned in Table 1, together with the atomic lines.

\section{Conclusions}

We observed the formation of the $o$-chlorobenzyl radical as an unexpected species as well as the 2,6-dichlorobenzyl radical as a typical product in a corona excited supersonic expansion from the corona discharge of 2,6-dichlorotoluene seeded in a large amount of inert carrier gas helium. The intramolecular reaction is a more feasible process for the replacement of chlorine atom by an adjacent hydrogen atom in the benzene ring because the reaction occurs in the gas phase. The mechanism for formation of the $o$-chlorobenzyl radicals in the gas phase has been proposed from the analysis of the spectrum.

Acknowledgments. This work was supported by Grant number R01-2008-000-20717-0 of the Basic Research Program of the Korea Science and Engineering Foundation and Korea Research Foundation Grant KRF-2008-314-C00161 from the Korean Government.

\section{References}

1. Carrington, A. Microwave Spectroscopy of Free Radicals; Academic: London, 1974.

2. Tan, X. Q.; Wright, T. G.; Miller, T. A. Electronic Spectroscopy of Free Radicals in Supersonic Jets: Jet Spectroscopy and Molecular Dynamics; Hollas, J. M., Phillip, D., Eds.; Blackie Academic \& Professional: London, 1994.

3. Selco, J. I.; Carrick, P. G. J. Mol. Spectrosc. 1989, 137, 13.

4. Lee, G. W.; Lee, S. K. Chem. Phys. Lett. 2009, 470, 54.

5. Lee, G. W.; Lee, S. K. J. Phys. Chem. A 2007, 111, 6003.

6. Lee, G. W.; Lee, S. K. J. Chem. Phys. 2007, 126, 214308.

7. Ahn, H. G.; Lee, G. W.; Kim, T. K.; Lee, S. K. Bull. Korean Chem. Soc. 2008, 29, 2341.

8. Ahn, H. G.; Lee, G. W.; Lee, S. K. J. Phys. Chem. A 2008, 112, 13427.

9. Lee, G. W.; Ahn, H. G.; Kim, T. K.; Lee, S. K. Chem. Phys. Lett. 2008, $465,193$. 
10. Ahn, H. G.; Lee, G. W.; Kim, T. K.; Lee, S. K. Chem. Phys. Lett. 2008, 454, 207.

11. Bindley, T. F.; Watts, A. T.; Walker, S. Trans. Faraday Soc. 1964, 60,1 .

12. Lee, S. K.; Baek, D. Y. Chem. Phys. Lett. 1999, 301, 407.

13. Lee, S. K.; Baek, D. Y. J. Phys. Chem. A 2000, 104, 5219.

14. Lee, S. K.; Ahn, B. U. Chem. Phys. Lett. 2000, 321, 25.

15. Lee, S. K.; Baek, D. Y. Chem. Phys. Lett. 1999, 311, 36.

16. Lee, S. K.; Baek, D. Y. Chem. Phys. Lett. 1999, 304, 39.

17. Lee, S. K.; Chae, S. Y. J. Phys. Chem. A 2001, 105, 5808.

18. Lee, S. K.; Chae, S. Y. J. Phys. Chem. A 2002, 106, 8054.

19. Fukushima, M.; Obi, K. Chem. Phys. Lett. 1996, 248, 269.

20. Lee, S. K.; Kim, S. J. Chem. Phys. Lett. 2005, 412, 88.
21. Lee, S. K. Chem. Phys. Lett. 2002, 358, 110.

22. Han, M. S.; Choi, I. S.; Lee, S. K. Bull. Korean Chem. Soc. 1996, 17, 991 .

23. Weise, M. L.; Smith, M. W.; Glennon, B. M. Atomic Transition Probabilities; NSRD -NBS4, 1966.

24. Cossart-Magos, C.; Cossart, D. Mol. Phys. 1988, 65, 627.

25. Banwell, C. N.; McCash, E. M. Fundamentals of Molecular Spectroscopy, 4th ed.; McGraw-Hill: London, 1994.

26. Fujiwara, M.; Tanimoto, Y. J. Phys. Chem. 1994, 98, 5695.

27. Suh, M. H.; Lee, S. K.; Rehfuss, B. D.; Miller, T. A.; Bondybey, V. E. J. Phys. Chem. 1991, 95, 2727.

28. Atkins, P. W. Physical Chemistry, 6th ed.; Oxford University Press: Oxford, 1998. 